\title{
A PROBLEM IN SHOGK WAVE DECAY
}

\author{
R. R. BURNSIDE and A. G. MACKIE \\ (received 27 October 1964)
}

\section{Introduction}

One of the elementary applications of the Rankine-Hugoniot shock relations which relate conditions on the two sides of a plane shock wave is that of determining the flow when a piston is pushed with constant velocity $\bar{u}$ into a tube containing gas at rest. A shock wave races into the undisturbed gas at a constant speed $\bar{U}$ whose value can easily be found in terms of $\bar{u}$ and the constants which specify the uniform condition of the gas at rest. If, however, the piston is suddenly brought to rest after a finite time the subsequent behaviour of the shock wave is very difficult to determine. A rarefaction wave is generated at the piston, and, as the velocity of the shock is subsonic relative to the gas behind it, this eventually overtakes the shock wave causing it to weaken. Since the energy supplied is finite the ultimate speed of the shock will tend to that of a sound wave. The analytical treatment of the flow behind the shock is made difficult by the entropy gradients which arise because of the variation in shock strength. It is further complicated by the disturbances which are reflected off the piston and give rise to a secondary interaction with the shock. Indeed, it seems safe to say that a complete description of the motion would certainly depend on some form of numerical integration.

For moderately weak shock strengths, the complete history of the shock can be determined approximately by means of a method due to Friedrichs [1]. This requires that we neglect the entropy variations behind the shock which are of the third order in the shock strength. To this degree of approximation the Riemann invariant

$$
\beta=-\frac{u}{2}+\frac{c}{\gamma-1}
$$

also remains constant through the shock. Here $u$ is the particle velocity, $c$ the local velocity of sound and $\gamma$ the adiabatic index of the gas. It follows that we may neglect the disturbances which are reflected back from the shock and that on this theory the path of the shock is determined from the Rankine-Hugoniot equations where the conditions in front of the shock are 
uniform and those behind are given by a point-centred simple wave. A refinement of this procedure was later given by Lighthill [3].

The method used in this paper does not limit in any way the strength of the shock but is applicable, on the other hand, only to the early stages of its decay. It consists of solving the system of first order equations obtained by perturbing the simple wave which interacts with the shock. Entropy variations are taken into account since they occur to the first order in the variation of the shock speed from its unperturbed value $\bar{U}$. An equation is found for the shock speed in the early stages of decay and in particular the initial deceleration of the shock can be determined. This agrees with that calculated from the work of Friedrichs for moderately weak shocks but is also applicable for strong shocks for which the results of Friedrichs are not valid. It also supplies some assessment of the range of shock strengths for which the approximation of Friedrichs is applicable.

\section{Statement of the Problem}

We shall work in the plane of $x$, the length coordinate along the tube and $t$, the time (Fig. 1). If the piston starts at $x=x_{L}, t=t_{L}$, where $x_{L}=\bar{u}_{L}$, then initially the equation of the shock is

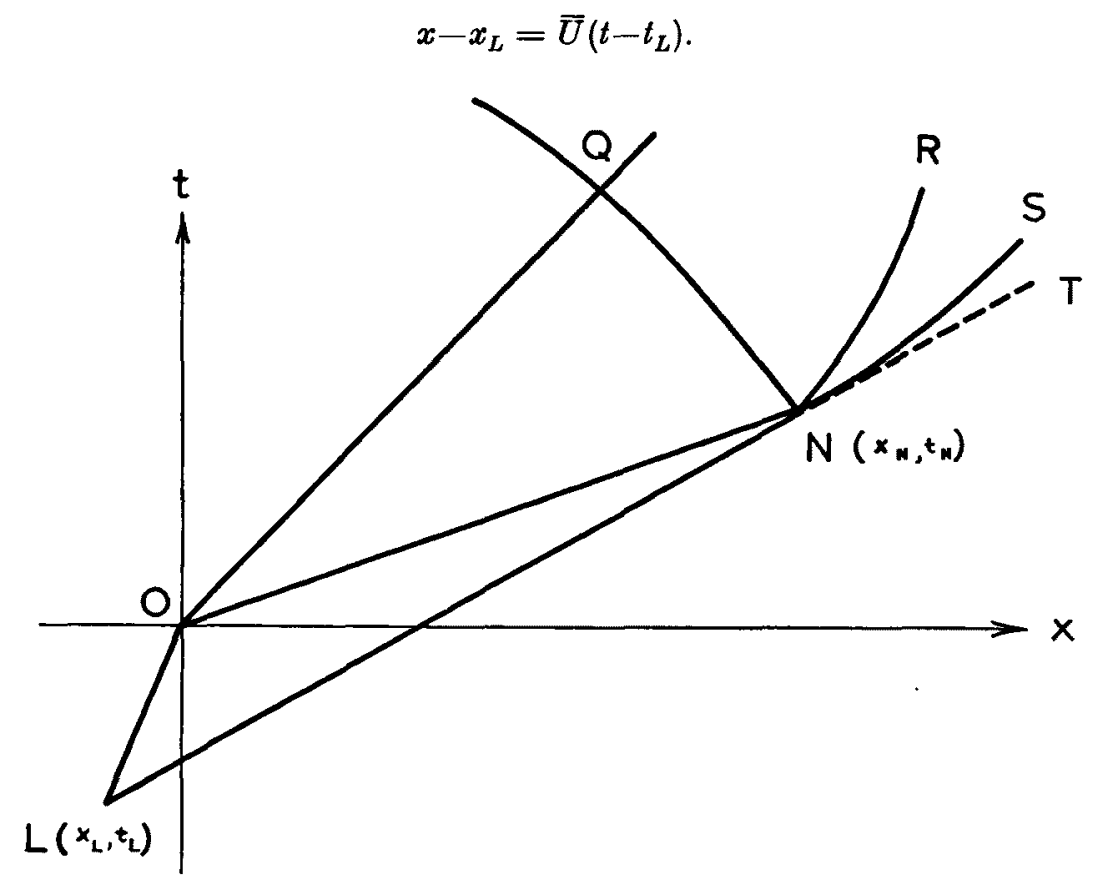

Fig. 1. 
If the piston is suddenly stopped at $x=0, t=0$, a point-centred simple wave $N O Q$ is generated at $O$ which first interacts with the shock wave at $N$. $N Q$ is the backward facing characteristic through $N$.

If a suffix 0 is used to denote the quantities in the simple wave and a bar to denote the (constant) quantities in the uniform region behind the shock, then

$$
\begin{aligned}
& u_{0}+c_{0}=\frac{x}{t}, \\
& -\frac{u_{0}}{2}+\frac{c_{0}}{\gamma-1}=\beta_{0}=-\frac{\bar{u}}{2}+\frac{\tilde{c}}{\gamma-1} .
\end{aligned}
$$

Thus

$$
\begin{aligned}
u_{0} & =\frac{2}{\gamma+1}\left[\frac{x}{t}-(\gamma-1) \beta_{0}\right], \\
c_{0} & =\frac{\gamma-1}{\gamma+1}\left[\frac{x}{t}+2 \beta_{0}\right],
\end{aligned}
$$

and the Riemann invariant $\alpha_{0}$, defined by

$$
\alpha_{0}=\frac{u_{0}}{2}+\frac{c_{0}}{\gamma-1},
$$

is given by

$$
\alpha_{0}=\frac{2}{\gamma+1}\left[\frac{x}{t}+\frac{3-\gamma}{2} \beta_{0}\right] .
$$

The shock path NS after the interaction has to be determined. For later convenience we note that in Fig. $1 N T$ is the straight line continuation of the undisturbed shock path $L N$.

The solution in the region QNS will be found on the basis of a first order perturbation of the simple wave. This region may be partitioned by $N R$, the particle path through $N$. Since the entropy remains constant on the particle paths, it follows that the entropy variations in the flow are confined to the region RNS. We have therefore four regions:

(i) $L O N$, a region of uniform flow,

(ii) $Q O N$, a point-centred simple wave with $u$ and $c$ given by (1) and (2),

(iii) $Q N R$, a simple wave plus an isentropic perturbation,

(iv) $R N S$, a simple wave plus a non-isentropic perturbation.

We now write down the governing equations and formulate the boundary value problem. If in the region QNS we let 


$$
u=u_{0}+u_{1}, c=c_{0}+c_{1}, \alpha=\alpha_{0}+\alpha_{1}, \beta=\beta_{0}+\beta_{1}, S=S_{0}+S_{1},
$$

where $S$ is the entropy and $S_{0}$ is a constant, then on substituting into the equations of one-dimensional unsteady flow and retaining only terms of the first order in the perturbation quantities which have suffix unity, we can write

$$
\frac{\partial \alpha_{1}}{\partial t}+\left(u_{0}+c_{0}\right) \frac{\partial \alpha_{1}}{\partial x}+\frac{1}{2}\left[(\gamma+1) \alpha_{1}-(3-\gamma) \beta_{1}\right] \frac{\partial \alpha_{0}}{\partial x}=\frac{c_{0}^{2}}{2 \gamma(\gamma-1) c_{v}} \frac{\partial S_{1}}{\partial x}
$$

$$
\frac{\partial \beta_{1}}{\partial t}+\left(u_{0}-c_{0}\right) \frac{\partial \beta_{1}}{\partial x}+\frac{1}{2}\left[(3-\gamma) \alpha_{1}-(\gamma+1) \beta_{1}\right] \frac{\partial \beta_{0}}{\partial x}=-\frac{c_{0}^{2}}{2 \gamma(\gamma-1) c_{v}} \frac{\partial S_{1}}{\partial x},
$$

$$
\frac{\partial S_{1}}{\partial t}+u_{0} \frac{\partial S_{1}}{\partial x}=0
$$

In these equations the constant $c_{v}$ is the specific heat at constant volume.

The above system of linear equations has three families of characteristics which are fixed curves in the $(x, t)$ plane, namely the $\alpha, \beta$ characteristics and the particle paths in the unperturbed simple wave. In particular, equation (5) expresses the fact that the entropy $S_{1}$ is constant along the particle paths of the unperturbed motion, given by

$$
\frac{d x}{d t}=u_{0}
$$

We may write the solution of equation (5) as

$$
S_{1}=S_{1}(y)
$$

where $y$ is some function of $x$ and $t$ which is constant on these particle paths. We shall define $y$ by

$$
y=c_{0}^{2} t^{2(\gamma-1) /(\gamma+1)},
$$

following Gundersen [2] who first obtained many of the results quoted in this section. In the same way, we shall later require another function $z(x, t)$, which is constant on the $\beta$-characteristics of the unperturbed flow. It is readily verified that

$$
z=\rho_{0} c_{0} t^{2}
$$

satisfies this requirement, $\rho_{0}(x, t)$ being the density of the fluid in the simple wave. The values of $y$ on $N R$ and $z$ on $N Q$ will be denoted respectively by $y_{n}$ and $z_{n}$.

The system (3) - (5) now reduces to 


$$
\begin{aligned}
& t \frac{\partial \alpha_{1}}{\partial t}+x \frac{\partial \alpha_{1}}{\partial x}+\alpha_{1}-\left(\frac{3-\gamma}{\gamma+1}\right) \beta_{1}=\frac{t c_{0}^{2} S_{1}^{\prime}(y)}{2 \gamma(\gamma-1) c_{v}} \frac{\partial y}{\partial x} \\
& t \frac{\partial \beta_{1}}{\partial t}+\left[\left(\frac{3-\gamma}{\gamma+1}\right) x-\frac{4 \beta_{0}(\gamma-1) t}{(\gamma+1)}\right] \frac{\partial \beta_{1}}{\partial x}=-\frac{t c_{0}^{2} S_{1}^{\prime}(y)}{2 \gamma(\gamma-1) c_{v}} \frac{\partial y}{\partial x}
\end{aligned}
$$

The solution in the isentropic region $Q N R$ will correspond to the complementary function of the above system while for the non-isentropic region RNS a particular integral will have to be added.

In terms of arbitrary functions the solutions are:

(8) (ii) Region $R N S, \alpha_{1}=\frac{3-\gamma}{\gamma+1} \frac{K(z)}{z^{\frac{1}{2}}}+\frac{1}{t} F\left(\frac{x}{t}\right)+\frac{\gamma+1}{\gamma-1} \frac{c_{0}}{y} \omega_{1}(y)$,

(i) Region $Q N R, \alpha_{1}=\frac{3-\gamma}{\gamma+1} \frac{H(z)}{z^{\frac{1}{2}}}+\frac{1}{t} G\left(\frac{x}{t}\right)$,

$$
\beta_{1}=2 z^{\frac{1}{2}} H^{\prime}(z) \text {. }
$$

$$
\beta_{1}=2 z^{\frac{1}{2}} K^{\prime}(z)+\frac{\gamma+1}{\gamma-1} \frac{c_{0}}{y} \omega_{1}(y) \text {. }
$$

The function $\omega_{1}(y)$ is related to $S_{1}(y)$ through the equation

$$
\omega_{1}(y)=\frac{1}{2 \gamma(\gamma+1) c_{n}} \int_{y_{n}}^{y} u S_{1}^{\prime}(u) d u+\text { constant. }
$$

It is easily shown that

$$
\frac{c_{0}}{y}=z^{-(\gamma-1) /(\gamma+1)}
$$

and hence that the constant of integration in (10) may be absorbed in the function $K(z)$, thus giving $\omega_{1}\left(y_{n}\right)=0$.

The boundary conditions to be satisfied by the general solution quoted above will now be formulated. If the equation of the shock locus is written

$$
x-x_{n}=\bar{U}\left(t-t_{n}\right)+\varepsilon(t),
$$

then $\varepsilon(t)$ represents the deviation from the straight line path. At the point $N$ we have

$$
\varepsilon\left(t_{n}\right)=\varepsilon^{\prime}\left(t_{n}\right)=0 .
$$

In what follows we shall neglect squares and higher powers of $\varepsilon(t)$ thus limiting attention to the initial stages of decay of the shock.

If we consider the values of $u$ and $c$ just behind the perturbed shock 
locus, then it is clear that they may be expressed in terms of $\bar{u}, \bar{c}$ and $\varepsilon^{\prime}(t)$. In fact

$$
\begin{aligned}
& u_{s}=\bar{u}+T_{1} \varepsilon^{\prime}(t), \\
& c_{s}=\bar{c}+T_{2} \varepsilon^{\prime}(t),
\end{aligned}
$$

where the suffix $s$ denotes conditions on the back of the shock and $T_{1}$, $T_{2}$ are known constants determined by the shock jump relations. Details are given in the Appendix. In addition, $\bar{u}$ and $\bar{c}$ are themselves related to the shock speed $\bar{U}$ and $C$, the velocity of sound in the region at rest ahead of the shock.

The values of $\alpha_{1}$ and $\beta_{1}$ at the shock, whose locus is given by (11), are

$$
\begin{aligned}
& \alpha_{18}=T_{3} \varepsilon^{\prime}(t)+\frac{2}{\gamma+1} \frac{\varepsilon(t)}{t}+\lambda(t), \\
& \beta_{1 s}=T_{4} \varepsilon^{\prime}(t),
\end{aligned}
$$

where

$$
\begin{aligned}
T_{3} & =\frac{T_{1}}{2}+\frac{T_{2}}{\gamma-1}, \quad T_{4}=-\frac{T_{1}}{2}+\frac{T_{2}}{\gamma-1} \quad \text { and } \\
\lambda(t) & =\frac{2}{\gamma+1}(\bar{u}+\bar{c}-\bar{U})\left(1-\frac{t_{n}}{t}\right) .
\end{aligned}
$$

Finally the entropy variations on the shock are given by

$$
S_{1 s}=T_{s} \varepsilon^{\prime}(t),
$$

where $T_{5}$ is a known constant (see Appendix).

This completes the boundary conditions on the shock locus NS. Those remaining are specified by continuity requirements along the characteristic $N Q$ which carries the front of the disturbance reflected back from the shock. They are

$$
\alpha_{1}=\beta_{1}=0 \text { when } z=z_{n} \text {. }
$$

Conditions (13), (14), (16) and (17) overspecify the boundary value problem. We therefore expect to obtain a relation between $\alpha_{1 s}, \beta_{1 s}$ and $S_{1 s}$ which will determine $\varepsilon(t)$.

\section{Solution of the Problem}

When the boundary condition (17) is used in the solution given by (6), then

$$
\frac{3-\gamma}{\gamma+1} \frac{H\left(z_{n}\right)}{z_{n}^{\frac{1}{2}}}+\left[\frac{1}{t} G\left(\frac{x}{t}\right)\right]_{z=z_{n}}=0 .
$$


Hence $G(x / t) \equiv 0$ and $H\left(z_{n}\right)=0$. Further $H^{\prime}\left(z_{n}\right)=0$ from (7). Thus, throughout the region $Q N R \alpha_{1}$ and $\beta_{1}$ are functions of $z$ only and are related by means of the equation

$$
2 z \frac{d \alpha_{1}}{d z}+\alpha_{1}=\frac{3-\gamma}{\gamma+1} \beta_{1}
$$

It is clear that the solutions for $\alpha_{1}$ and $\beta_{1}$ in the non-isentropic region $R N S$ must satisfy the relationship (18) along the curve $N R$ where $d \alpha_{1} / d z$ is now interpreted as differentiation with respect to $z$ along $N R$. Thus from (8) and (9), we have on $N R$

$$
\begin{gathered}
\frac{d}{d z}\left[K(z)+\frac{\gamma+1}{3-\gamma} \frac{z^{\frac{1}{2}}}{t} F\left(\frac{x}{t}\right)+\frac{(\gamma+1)^{2}}{(\gamma-1)(3-\gamma)} \frac{c_{0} z^{\frac{1}{2}}}{y} \omega_{1}(y)\right] \\
=K^{\prime}(z)+\frac{\gamma+1}{\gamma-1} \frac{c_{0}}{y z^{\frac{1}{2}}} \omega_{1}\left(y_{n}\right) .
\end{gathered}
$$

Since $\omega_{1}\left(y_{n}\right)=0$, it follows that $F(x / t) \equiv 0$.

When the solutions for $\alpha_{1}$ and $\beta_{1}$ in the isentropic and non-isentropic regions are considered it is clear that the functions $H(z)$ and $K(z)$ must be identical. Physically this ensures that the flow is continuous across $N R$. Thus

(i) Region $Q N R, \quad \alpha_{1}=\frac{3-\gamma}{\gamma+1} \frac{K(z)}{z^{\frac{1}{2}}}$,

$$
\beta_{1}=2 z^{\frac{1}{2}} K^{\prime}(z) \text {. }
$$

(ii) Region $R N S, \quad \alpha_{1}=\frac{3-\gamma}{\gamma+1} \frac{K(z)}{z^{\frac{1}{z}}}+\frac{\gamma+1}{\gamma-1} \frac{c_{0}}{y} \omega_{1}(y)$,

$$
\beta_{1}=2 z^{\frac{1}{2}} K^{\prime}(z)+\frac{\gamma+1}{\gamma-1} \frac{c_{0}}{y} \omega_{1}(y) .
$$

It follows from the above that the perturbation of the particle velocity $u_{1}$ is unaffected by the variations in entropy.

The solution in the region RNS must satisfy the boundary conditions (13) and (14) on the shock locus NS. Moreover, the entropy $S_{1}$, which is connected to $\omega_{1}(y)$ by means of (10), must satisfy (16) on $N S$. We first of all eliminate $K(z)$ from (19) and (20) by differentiating along the shock locus and obtain, after substituting for $\omega_{1}(y)$ in terms of $S_{1}$, the relation

$$
\frac{d \alpha_{1 s}}{d t}-\frac{c_{0 s}}{2 \gamma(\gamma-1) c_{v}} \frac{d S_{1 s}}{d t}+\frac{1}{2} \frac{d \log z_{s}}{d t}\left[\alpha_{1 s}-\frac{3-\gamma}{\gamma+1} \beta_{1 s}\right]=0 \text {. }
$$

If in this equation we substitute for $\alpha_{1 s}, \beta_{1 s}$ and $S_{18}$ in terms of $\varepsilon(t)$ and retain only terms of the first order, then 


$$
\begin{aligned}
{\left[T_{3}-\right.} & \left.\frac{c_{0 s} T_{5}}{2 \gamma(\gamma-1) c_{v}}\right] \varepsilon^{\prime \prime}(t)+\frac{1}{2}\left[\frac{2}{t}+\frac{\gamma+1}{\gamma-1} \frac{d \log c_{0 s}}{d t}\right]\left[T_{3}-\frac{3-\gamma}{\gamma+1} T_{4}\right] \varepsilon^{\prime}(t) \\
& +\left[\frac{2}{\gamma+1}+\frac{\lambda(t)}{2 c_{0 s}}\right] \frac{d}{d t}\left\{\frac{\varepsilon(t)}{t}\right\}+\frac{1}{2}\left[\frac{4}{\gamma+1} \frac{1}{t}-\frac{2}{\gamma-1} \frac{d \log c_{0 s}}{d t}\right. \\
& \left.-\frac{\lambda(t)}{c_{0 s}} \frac{d \log c_{0 s}}{d t}\right] \frac{\varepsilon(t)}{t} \\
& =-\lambda^{\prime}(t)-\frac{\lambda(t)}{2}\left[\frac{2}{t}+\frac{\gamma+1}{\gamma-1} \frac{d \log c_{0 s}}{d t}\right] .
\end{aligned}
$$

The terms involving the quantity $\varepsilon(t) / t$ arise from applying the boundary conditions to the shock locus $N S$, rather than to the straight line path $N T$. In equation (22) we note that

$$
c_{0 s}=\bar{c}-\frac{\gamma-1}{2} \lambda(t) .
$$

Thus we have a second order linear differential equation for $\varepsilon(t)$ with rational functions of $t$ as coefficients. The equation is to be solved for values of $t \geqq t_{n}$ with (12) as boundary conditions.

Since only linear terms in $\varepsilon(t)$ are retained in (22), this equation can be regarded as valid only in the initial stages of decay. Rather than give its full solution, we merely calculate the successive derivatives of $\varepsilon(t)$ at the point $N$. In this manner a Taylor series expansion of $\varepsilon(t)$ for $t \geqq t_{n}$ is constructed. In view of (12) the initial term of this series is $\frac{1}{2} \varepsilon^{\prime \prime}\left(t_{n}\right)\left(t-t_{n}\right)^{2}$ and $\varepsilon^{\prime \prime}\left(t_{n}\right)$, obtained from $(22)$, is given by

$$
\left[T_{3}-\frac{\bar{c} T_{5}}{2 \gamma(\gamma-1) c_{v}}\right] \varepsilon^{\prime \prime}\left(t_{n}\right)=-\lambda^{\prime}\left(t_{n}\right) .
$$

We note that terms of (22) involving $\varepsilon(t) / t$ will contribute to $\varepsilon^{\prime \prime \prime}\left(t_{n}\right)$ and all higher derivatives.

If we regard the initial stages of the shock locus as now known, then the functions $K(z)$ and $\omega_{1}(y)$ may be determined. The complete solution is valid within the band-like region bounded by the relevant portion of the shock locus, the $\beta$-characteristic on which $z=z_{n}$, the continuation of the $\alpha$-characteristic which bounds the left hand end of the simple wave and a $\beta$-characteristic whose position is dependent on the order of the Taylor series expansion which is used for $\varepsilon(t)$.

\section{Discussion of the Solution}

One would expect some association between the present theory and that of Friedrichs [1]. The Friedrichs theory is recovered by assuming 
the Riemann invariant $\beta$ and the entropy $S$ to be constant with their values specified by the uniform conditions ahead of the shock wave. This means that we take $S_{1} \equiv 0$ and $\beta_{1} \equiv 0$ and equation (21) then reduces to

$$
\frac{d \alpha_{1 s}}{d t}+\frac{1}{2} \frac{d \log z_{s}}{d t} \alpha_{1 s}=0,
$$

with $\alpha_{18}=0$ when $t=t_{n}$.

The appropriate solution of this equation is $\alpha_{18}=0$ and so

$$
\alpha_{s}=\alpha_{0 s} \text { when } t \geqq t_{n} .
$$

From this relation, the results of the "simple wave" approximation of Friedrichs may be derived. Thus the first order perturbation theory reduces to the well-known theory of Friedrichs when the appropriate assumptions are made.

One interesting physical quantity coming out of the present work is the initial deceleration of the shock wave $\varepsilon^{\prime \prime}\left(t_{n}\right)$. It is of interest to compare the values of this quantity as predicted by the present method and that of Friedrichs.

From (15), it follows that

$$
\lambda^{\prime}(t)=\frac{2(\bar{u}+\bar{c}-\bar{U})}{(\gamma+1) t_{n}} .
$$

The Rankine-Hugoniot shock relations enable all quantities of (23) involving $u$ and $c$ to be expressed in terms of a Mach number $M$ defined by

$$
M=\frac{\bar{U}}{C} \quad(1 \leqq M<\infty) .
$$

The following expression for $\varepsilon^{\prime \prime}\left(t_{n}\right)$ is then found, the details of the derivation being indicated in the Appendix.

$$
\begin{aligned}
& \varepsilon^{\prime \prime}\left(t_{n}\right) \\
& =-\frac{2}{(\gamma+1)} \frac{C}{t_{n}}\left[(\gamma-1) M^{2}+2\right]^{\frac{1}{2}}\left[\frac{\sqrt{2 \gamma M^{2}-(\gamma-1)}-\sqrt{(\gamma-1) M^{2}+2}}{1+M^{2}+2 M^{2} \sqrt{\frac{(\gamma-1) M^{2}+2}{2 \gamma M^{2}-(\gamma-1)}}}\right] .
\end{aligned}
$$

We observe that for very weaks shock $\varepsilon^{\prime \prime}\left(t_{n}\right) \rightarrow 0$ as $M \rightarrow 1$ as would be expected, whilst for very strong shocks

$$
\varepsilon^{\prime \prime}\left(t_{n}\right) \rightarrow-\frac{2}{\gamma+1} \frac{C}{t_{n}} \frac{2 \gamma \sqrt{(\gamma-1)}-(\gamma-1) \sqrt{(2 \gamma)}}{\sqrt{(2 \gamma)}+2 \sqrt{(\gamma-1)}} \text { as } M \rightarrow \infty .
$$

The initial deceleration of the shock wave as calculated * from the Friedrichs

* The authors accept the responsibility for the accuracy of $(26)$ which is not quoted by Friedrichs [1]. 
theory is derived in the Appendix and is

$$
\varepsilon^{\prime \prime}\left(t_{n}\right)=\left(\frac{d \dot{\xi}}{d t}\right)_{n}=-\frac{C}{2 t_{n}} \delta\left[1+O\left(\delta^{2}\right)\right],
$$

where $d x=\xi d t$ represents the shock path and $\delta=M-1$. The corresponding expansion of (25) to a higher order in $\delta$ is

$$
\varepsilon^{\prime \prime}\left(t_{n}\right)=-\frac{C}{2 t_{n}} \delta\left[1-\frac{\delta^{2}}{4}+O\left(\delta^{3}\right)\right] .
$$

In Table 1 some numerical values of the initial deceleration of the shock wave as predicted by (25), (26) and (27) are shown for a range of $\delta \leqq 0.7$ with the adiabatic index $\gamma=1.4$.

TABLE 1

\begin{tabular}{cccc}
\hline$\delta$ & $\Delta_{1}$ & $\Delta_{\text {? }}$ & $\Delta_{3}$ \\
\hline 0.1 & 0.050 & 0.050 & 0.050 \\
0.2 & 0.100 & 0.100 & 0.100 \\
0.3 & 0.150 & 0.146 & 0.147 \\
0.4 & 0.200 & 0.192 & 0.194 \\
0.5 & 0.250 & 0.234 & 0.239 \\
0.6 & 0.300 & 0.273 & 0.280 \\
0.7 & 0.350 & 0.307 & 0.320 \\
\hline
\end{tabular}

$\Delta_{1}, \Delta_{2}, \Delta_{3}$ respectively represent the values of $-t_{n} \varepsilon^{\prime \prime}\left(t_{n}\right) / C$ as calculated from the "simple wave" approximation (26), equation (27) and the exact solution (25).

On the "simple wave" approximation, the initial deceleration of the shock wave is a linear function of $\delta$ and we observe that this theory overestimates the rate at which the velocity of the shock wave diminishes at the initial point of decay. However, even when $\delta$ has as large a value as 0.7 , corresponding to a pressure ratio of $\mathbf{2 . 6 7 5}$, the solution from the "simple wave" approximation differs from (25) by less than $10 \%$. The corresponding difference in the solutions as given by (25) and (27) is $4 \%$. Equation (27) includes terms which are of the order of the cube of the shock strength and it is interesting to note that the difference between (27) and (26) is independent of the adiabatic index of the gas.

We conclude by quoting expressions for $\alpha_{1 s}$ and $\beta_{1 s}$ valid in the neighbourhood of the initial point of decay of the shock. The derivation of those results is indicated in the Appendix.

$$
\begin{aligned}
& \alpha_{18}=\left[\Gamma_{1} \tau^{2}+O\left(\tau^{3}\right)\right]+\left\{\Gamma_{2} \tau+O\left(\tau^{2}\right)\right\}, \\
& \beta_{1 s}=\left[\Gamma_{3} \tau+O\left(\tau^{2}\right)\right]+\left\{\Gamma_{2} \tau+O\left(\tau^{2}\right)\right\},
\end{aligned}
$$


where $\tau=t / t_{n}-1$, with $t$ denoting time measured on the shock path, and the equations have been written in this particular way to facilitate later discussion and analysis. The quantities $\Gamma_{1}, \Gamma_{2}$ and $\Gamma_{3}$ are given by

$$
\begin{aligned}
& \Gamma_{1}=\frac{3-\gamma}{2(\gamma+1)} z_{n}^{3} K^{\prime \prime}\left(z_{n}\right)\left[\frac{\bar{U}+\bar{c}-\bar{u}}{\bar{c}}\right]^{2}, \\
& \Gamma_{2}=\frac{T_{5} \varepsilon^{\prime \prime}\left(t_{n}\right) t_{n} \bar{c}}{2 \gamma(\gamma-1) c_{n}}, \\
& \Gamma_{3}=2 z_{n}^{\sharp} K^{\prime \prime}\left(z_{n}\right)\left[\frac{\bar{U}+\bar{c}-\bar{u}}{\bar{c}}\right]
\end{aligned}
$$

with

$$
2 z_{n}^{\prime \prime} K^{\prime \prime}\left(z_{n}\right)=-\frac{\bar{c} t_{n}}{\bar{U}+\bar{c}-\bar{u}}\left[\lambda^{\prime}\left(t_{n}\right)+\left(T_{3}-T_{4}\right) \varepsilon^{\prime \prime}\left(t_{n}\right)\right]
$$

The terms within the square brackets in (28) and (29) are derived from those of (19) and (20) which involve $K\left(z_{s}\right)$ and its derivative, whilst the terms in the curly brackets are derived from those involving $\omega_{1}\left(y_{s}\right)$.

No approximations have been used in (28) and (29) other than those inherent in the Taylor series expansion. To this approximation (28) and (29) are valid when $\tau$ is sufficiently small for a shock wave of any initial strength. In (28), we note that the leading term in the square bracket is of a higher order of magnitude in $\tau$ than that given by the curly bracket. This suggests that the contribution to $\alpha_{1 s}$ carried along the particle paths is more important (at least for points sufficiently close to $N$ ) than that which is carried by the $\beta$-characteristics. On the other hand, (29) indicates that the contributions carried by the particle paths and the $\beta$-characteristics are of the same order in $\tau$.

For weak shock waves we may expand the coefficients $\Gamma_{1}, \Gamma_{2}, \Gamma_{3}$ in terms of $\delta=M-1$ to terms of order the cube of the shock strength. We then obtain

$$
\begin{aligned}
& \Gamma_{1}=-C \frac{(3-\gamma)(3 \gamma-5)}{16(\gamma+1)^{3}} \delta^{3}+\cdots \\
& \Gamma_{2}=-C \frac{4}{(\gamma+1)^{2}} \delta^{3}+\cdots \\
& \Gamma_{3}=-C \frac{(3 \gamma-5)}{2(\gamma+1)^{2}} \delta^{3}+\cdots
\end{aligned}
$$

In the special case when $\gamma=5 / 3$, we note that $\Gamma_{1}=0=\Gamma_{3}$ and consequently $\alpha_{1 s}$ and $\beta_{1 s}$ depend only on the variations in entropy. To this approximation, there is no reflected wave. For air with $\gamma=7 / 5$ we obtain 


$$
\Gamma_{1}: \Gamma_{2}: \Gamma_{3}=1:-120: 12 \text {. }
$$

Thus for the above values of $\gamma$ the major contribution to $\alpha_{1 s}$ and $\beta_{1 s}$ comes from the entropy variations. The reflected wave would seem to play a secondary role. The results indicate why the Friedrichs theory is more accurate than one would perhaps at first expect. Of some interest also is the fact that shock-expansion theory (Pillow [5], Meyer [4]) is based on the relative importance of the role played by the particle paths, as the present theory emphasizes.

\section{Acknowledgement}

One of the authors (R.R.B.) wishes to express his gratitude to the Department of Scientific and Industrial Research in the United Kingdom for the Research Studentship which he held whilst engaged on this work.

\section{Appendix}

1. The constants $T_{1}, T_{2}, T_{3}, T_{4}, T_{5}$.

The path of the shock in the physical plane is given by $d x=\dot{\xi} d t$. If $M=\dot{\xi} / C$, then the Rankine-Hugoniot shock relations are

$$
\begin{aligned}
& u=\frac{2 C\left(M^{2}-1\right)}{(\gamma+1) M}, \\
& c=\frac{C}{(\gamma+1) M} \sqrt{ }\left\{(\gamma-1) M^{2}+2\right\}\left\{2 \gamma M^{2}-(\gamma-1)\right\}, \\
& \frac{S-S_{0}}{c_{v}}=\log \left[\left\{2 \gamma M^{2}-(\gamma-1)\right\}\left\{\frac{(\gamma-1) M^{2}+2}{M^{2}}\right\}^{\gamma}\right] .
\end{aligned}
$$

If the above relations are differentiated with respect to $\dot{\xi}$, then

$$
\begin{aligned}
& T_{1} \equiv \frac{d u}{d \dot{\xi}}=\frac{2\left(M^{2}+1\right)}{(\gamma+1) M^{2}} \\
& T_{2} \equiv \frac{d c}{d \dot{\xi}}=\frac{2(\gamma-1)}{\gamma+1}\left[\frac{\gamma M^{4}+1}{M^{2} \sqrt{ }\left\{2 \gamma M^{2}-(\gamma-1)\right\}\left\{(\gamma-1) M^{2}+2\right\}}\right] \\
& T_{5} \equiv \frac{d S}{d \dot{\xi}}=\frac{4 \gamma(\gamma-1) c_{v}}{C}\left[\frac{\left(1-M^{2}\right)^{2}}{M\left\{2 \gamma M^{2}-(\gamma-1)\right\}\left\{(\gamma-1) M^{2}+2\right\}}\right] .
\end{aligned}
$$

The constants $T_{1}, T_{2}, T_{3}, T_{4}, T_{5}$ noted in $\S 2$ are obtained from the above with $M=\bar{U} / C$. 
2. Expansion of $T_{3}, T_{4}, T_{5}$ in terms of $\delta=M-1$.

From the above, we have, for $\delta<0.622$,

$$
\begin{aligned}
& T_{1}=\frac{4}{\gamma+1}\left[1-\delta+\frac{3}{2} \delta^{2}+O\left(\delta^{3}\right)\right] \\
& T_{2}=\frac{2(\gamma-1)}{\gamma+1}\left[1-\delta+3 \delta^{2}+O\left(\delta^{3}\right)\right] .
\end{aligned}
$$

Hence

$$
T_{3}=\frac{T_{1}}{2}+\frac{T_{2}}{\gamma-1}=\frac{4}{\gamma+1}\left[1-\delta+\frac{9}{4} \delta^{2}+O\left(\delta^{3}\right)\right]
$$

and

$$
T_{4}=-\frac{T_{1}}{2}+\frac{T_{2}}{\gamma-1}=\frac{3}{\gamma+1} \delta^{2}[1+O(\delta)]
$$

Also,

and

$$
T_{5}=\frac{16 \gamma(\gamma-1) c_{v}}{(\gamma+1)^{2} C} \delta^{2}[1+O(\delta)]
$$

$$
\bar{u}+\bar{c}-\bar{U}=C \delta\left[1-\delta+\frac{2 \gamma}{\gamma+1} \delta^{2}+O\left(\delta^{3}\right)\right] .
$$

3. The initial deceleration of the shock wave according to the simple reave approximation.

On differentiating (24) with respect to time $t$, measured along the shock locus, we obtain

$$
\frac{d \alpha_{s}}{d \dot{\xi}} \frac{d \dot{\xi}}{d t}=-\frac{2}{\gamma+1}\left[\frac{(u+c-U)}{t^{2}} t_{n}\right]
$$

But $d \alpha_{3} / d \xi=T_{3}$ and from section 2 of this Appendix

$$
T_{3}=\frac{4}{\gamma+1}\left[1-\delta+O\left(\delta^{2}\right)\right]
$$

the expansion being valid to the order implied by the approximation. To the same order,

$$
\bar{u}+\bar{c}-\bar{U}=C \delta\left[1-\delta+O\left(\delta^{2}\right)\right] .
$$

Hence, at the initial point of decay of the shock wave,

$$
\left(\frac{d \dot{\xi}}{d t}\right)_{n}=-\frac{C}{2 t_{n}} \delta\left[1+O\left(\delta^{2}\right)\right]
$$


4. Determination of $K\left(z_{s}\right)$ and $\omega_{1}\left(y_{s}\right)$ as functions of $\tau$.

On taking (19), (20) at the shock locus and differentiating with respect to time $t$ measured along the shock locus and then subtracting and using boundary conditions (13) and (14), we obtain the following relation at the initial point $N$ :

$$
2 z_{n}^{\frac{1}{2}} K^{\prime \prime}\left(z_{n}\right)=-\left[\lambda^{\prime}\left(t_{n}\right)+\left(T_{3}-T_{4}\right) \varepsilon^{\prime \prime}\left(t_{n}\right)\right] /\left(\frac{d z_{s}}{d t}\right)_{n} .
$$

However, it is readily shown that

$$
\left(\frac{d z_{s}}{d t}\right)_{n}=\frac{z_{n}}{t_{n}}\left[\frac{\bar{U}+\bar{c}-\bar{u}}{\bar{c}}\right] .
$$

Hence

$$
2 z_{n}^{\prime} K^{\prime \prime}\left(z_{n}\right)=-\frac{\bar{c} t_{n}\left[\lambda^{\prime}\left(t_{n}\right)+\left(T_{3}-T_{4}\right) \varepsilon^{\prime \prime}\left(t_{n}\right)\right]}{\bar{U}+\bar{c}-\bar{u}} .
$$

But

$$
K\left(z_{s}\right)=\frac{1}{2} K^{\prime \prime}\left(z_{n}\right)\left(z_{s}-z_{n}\right)^{2}+O\left(z_{s}-z_{n}\right)^{3}
$$

and it is easily shown that

$$
z_{s}-z_{n}=z_{n}\left[\frac{\bar{U}+\bar{c}-\bar{u}}{\bar{c}}\right] \tau+O\left(\tau^{2}\right) .
$$

Hence

$$
K\left(z_{s}\right)=\frac{1}{2} z_{n}^{2} K^{\prime \prime}\left(z_{n}\right)\left[\frac{\bar{U}+\bar{c}-\bar{u}}{\bar{c}}\right]^{2} \tau^{2}+O\left(\tau^{3}\right)
$$

and

$$
K^{\prime}\left(z_{s}\right)=z_{n} K^{\prime \prime}\left(z_{n}\right)\left[\frac{\bar{U}+\bar{c}-\bar{u}}{\bar{c}}\right] \tau+O\left(\tau^{2}\right)
$$

From (20),

with

$$
\beta_{18}=T_{4} \varepsilon^{\prime}(t)=2 z_{s}^{\frac{1}{s}} K^{\prime}\left(z_{s}\right)+\frac{\gamma+1}{\gamma-1} \frac{c_{0 s}}{y_{s}} \omega_{1}\left(y_{s}\right),
$$

and

$$
\omega_{1}\left(y_{s}\right)=\omega_{1}^{\prime}\left(y_{n}\right)\left(y_{s}-y_{n}\right)+O\left(y_{s}-y_{n}\right)^{2}
$$

$$
\omega_{1}^{\prime}\left(y_{n}\right)=\frac{y_{n} S_{1}^{\prime}\left(y_{n}\right)}{2 \gamma(\gamma+1) c_{v}} .
$$

Hence

$$
\omega_{1}^{\prime}\left(y_{n}\right)=\frac{y_{n} T_{5} \varepsilon^{\prime \prime}\left(t_{n}\right)}{2 \gamma(\gamma+1) c_{v} y_{a}^{\prime}\left(t_{n}\right)}
$$


Accordingly,

$$
\frac{\gamma+1}{\gamma-1} \frac{c_{08}}{y_{s}} \omega_{1}\left(y_{s}\right)=\frac{T_{5} \varepsilon^{\prime \prime}\left(t_{n}\right)}{2 \gamma(\gamma-1) c_{v}} \frac{c_{08} y_{n}}{y_{s} y_{s}^{\prime}\left(t_{n}\right)}\left(y_{s}-y_{n}\right)+O\left(y_{s}-y_{n}\right)^{2}
$$

But

$$
\begin{aligned}
& \frac{y_{s}}{y_{n}}=1+2\left(\frac{\gamma-1}{\gamma+1}\right)\left[\frac{\bar{U}-\bar{u}}{\bar{c}}\right] \tau+O\left(\tau^{2}\right), \\
& \frac{c_{0 s}}{\bar{c}}=1-\left(\frac{\gamma-1}{\gamma+1}\right)\left[\frac{\bar{U}+\bar{c}-\bar{u}}{\bar{c}}\right] \tau+O\left(\tau^{2}\right)
\end{aligned}
$$

and

$$
\left(\frac{y_{s}^{\prime}}{y_{n}}\right)_{n}=\frac{2(\gamma-1)}{t_{n}(\gamma+1)}\left[\frac{\bar{U}-\bar{u}}{\bar{c}}\right]
$$

Hence, in (A.3) we obtain

$$
\frac{\gamma+1}{\gamma-1} \frac{c_{0 s}}{y_{s}} \omega_{1}\left(y_{s}\right)=\frac{T_{5} \bar{c} t_{n} \varepsilon^{\prime \prime}\left(t_{n}\right)}{2 \gamma(\gamma-1) c_{v}} \tau+O\left(\tau^{2}\right) .
$$

From (A.1), (A.2) and (A.4), equations (28) and (29) are easily obtained.

\section{References}

[1] Friedrichs, K. O., Formation and decay of shock waves, Comm. Pure Appl. Math. 1 (1948), $211-245$.

[2] Gundersen, R., The flow of a compressible fluid with weak entropy changes, $J$. Fluid Mech. 3 (1958), 553-581.

[3] Lighthill, M. J., The energy distribution behind decaying shocks, Phil. Mag. 41 (1950), $1101-1128$.

[4] Meyer, R. E., The analytical theory of characteristics of inviscid gas dynamics, Techn. Rep. No. 24, Div. Appl. Math. Brown University (1959).

[5] Pillow, A. F., The formation and growth of shock waves in the one-dimensional motion of a gas, Proc. Camb. Phil. Soc. 45 (1949), 558-586.

University of Toronto, Canada.

Victoria University of Wellington, New Zealand. 